\title{
Application of in situ simulation teaching in the training of trainee nurses to respond to emergencies
}

\author{
Wen Wang ${ }^{1 \#}$, Chaojuan Han ${ }^{2 \#}$, Xinhui Zhang ${ }^{1}$, Yeying Tong ${ }^{1}$, Ruxia Zhao ${ }^{3}$, Bin Wang ${ }^{3}$, Wei Xing ${ }^{2}$, \\ Ni Wang ${ }^{4}$, Chujun Chen ${ }^{4}$ \\ ${ }^{1}$ Department of Pediatrics, the First Affiliated Hospital of Hainan Medical University, Haikou, China; ${ }^{2}$ Department of Obstetrics, Haikou Hospital \\ of the Maternal and Child Health, Haikou, China; ${ }^{3}$ Department of Pharmacy, Haikou Hospital of the Maternal and Child Health, Haikou, China; \\ ${ }^{4}$ Department of General surgery, Tunchang People's Hospital, Tunchang, China \\ Contributions: (I) Conception and design: W Wang, C Han, X Zhang; (II) Administrative support: W Wang, Y Tong, R Zhao; (III) Provision of study \\ materials or patients: B Wang, W Xing, W Wang; (IV) Collection and assembly of data: All authors; (V) Data analysis and interpretation: W Wang, \\ N Wang, C Chen; (VI) Manuscript writing: All authors; (VII) Final approval of manuscript: All authors. \\ \#These authors contributed equally to this work. \\ Correspondence to: Chujun Chen. Department of General Surgery, Tunchang People's Hospital, Tunchang 571600, China. \\ Email: chenchujun3930@163.com.
}

Background: To explore the effect of the in situ simulation teaching method in the emergency training of trainee nurses.

Methods: A total of 108 trainee nurses from the First Affiliated Hospital of Hainan Medical College were selected, and in situ simulation teaching was employed in emergency training. Following the in situ simulation teaching training, a questionnaire was issued to evaluate CIPP (context evaluation, input evaluation, process evaluation, product evaluation) simulation teaching, clinical thinking ability, nursing team cooperation, and student satisfaction. These data were then collected and statistical analysis was conducted.

Results: The response rate of this teaching questionnaire was $100 \%$. After using in situ simulation teaching to instruct trainee nurses in emergencies, the satisfaction rate of the trainee nurses was $94.9 \%$, and the satisfaction rate of the instructor with the trainee nurses was $92.2 \%$. After in-situ simulation teaching, the clinical thinking ability (critical thinking ability, systematic thinking ability, evidence-based thinking ability), teamwork ability (trust, team orientation, support, shared mental model and team leadership), the theoretical and clinical practice ability had been improved.

Conclusions: Most nurses agreed that the in situ simulation teaching method can cultivate clinical thinking and teamwork ability for common emergencies, thereby improving their comprehensive quality and job competence, which is invaluable when responding to emergencies.

Keywords: In situ simulation teaching; emergency training; clinical application

Submitted Feb 05, 2021. Accepted for publication Apr 01, 2021.

doi: $10.21037 /$ apm-21-545

View this article at: http://dx.doi.org/10.21037/apm-21-545

\section{Introduction}

In situ simulation involves placing an emergency care simulator (ESC) simulator in an actual clinical working environment (such as a ward, toilet, corridor, etc.); that is, instead of simulating the working scene, in situ simulation involves restoring the clinic and being present in the actual working scene. This may help to deepen the understanding of nurses in a real and intuitive way, and thereby improve the teaching effect (1). Simulation teaching has established an important position in medical education, but according to existing research and literature at home and abroad, simulation content is primarily available in the fields of anesthesia, first aid, and critical illness (2). However, there are few reports on simulation teaching courses with nursing 
emergency incidents as the main content, especially in situ simulation teaching (3). Traditional theoretical teaching can only educate students about the corresponding knowledge points, and cannot enable students to fully and proficiently master clinical thinking and processing procedures, and cannot meet the requirements of the new model of medical education. Therefore, the in situ simulation teaching method, which is used to improve the clinical thinking and teamwork ability of nurses in emergency nursing, as well as supplement the lack of nursing and clinical thinking in the field, forms an important part of simulation teaching to cultivate practical nursing talents. We present the following article in accordance with the MDAR reporting checklist (available at http://dx.doi.org/10.21037/apm-21-545).

\section{Methods}

\section{General information}

In 2019, 108 trainee nurses from the First Affiliated Hospital of Hainan Medical University were selected as the experimental group, all of whom were females with an average age of 23 years. In addition, 108 nurses who had worked for more than 5 years were selected as the control group, all of whom were female, and with an average age of 29 years. The study was conducted in accordance with the Declaration of Helsinki (as revised in 2013). The study protocol was approved by the ethics committee of the First Affiliated Hospital of Hainan Medical University (No. 20190232). Informed consent was taken from all participants.

\section{Designation of in situ simulation teaching courses}

Issues related to emergencies were selected for in situ simulation, such as sudden changes in the condition of a patient (postoperative bleeding, shock, asphyxia, cardiac arrest, etc.), adverse events (bed falling/falling, serious adverse drug reactions, etc.), and equipment failure.

\section{Special lectures}

Spiral teaching was used to estimate and guide common problems in in situ simulation practice.

\section{Data preparation}

The patient's general information, chief complaint, current medical history, and types of emergencies were sent to the nurses. The nurses consulted relevant literature and books based on the data, learned the theoretical knowledge, and prepared simulated teaching courses in the form of flipped classrooms.

\section{Scene simulation}

The simulation was conducted as follows: the clinical nursing ward was selected, and the existing monitoring facilities, rescue items, basic nursing operations, and other supplies were utilized, as well as the emergency care simulator (ECS) simulator system for layout. The scene should make the nurses feel like they are in an accurate representation of an actual situation, and provide a good experience effect. Basically, a group of four nurses took turns as the responsible nurse, with the rest as auxiliary nurses. The instructor was responsible for controlling the ECS system. The voice system could be used for dialogue and communication between patients and nurses, and information technology (IT) engineers could also provide assistance. At the same time, a doctor was invited to participate in the simulated teaching activities. The on-duty nurse began by checking the patient's vital signs (according to the handling processes of different emergency events), asking the patient about their primary complaint, observing their condition, and performing the corresponding physical examinations. Group discussion based on medical history, physical signs, laboratory and auxiliary examination results was conducted in order to make a nursing diagnosis. This information, combined with the specialized theoretical knowledge and clinical nursing principles that have been learned, were utilized by the team members to implement emergency treatment on patients. The teacher responded to the nurse's treatment measures verbally or on the monitor by controlling the simulation system. According to the curriculum design, the teacher presented the patients with various emergencies. The nurses constantly selected and adjusted nursing measures in a targeted manner based on patient responses and changes in vital signs. If the treatment and nursing measures are applied correctly, the patient (advanced simulator) turned from danger to safety, which signified that the nurse's judgment was correct. However, improper or untimely selection of treatment and nursing measures affected the rescue effect, delayed the rescue opportunity, and in severe cases led to the death of the patient (senior simulator).

\section{Analysis and summary}

Simulated teaching was performed using the CIPP (Context 
Table 1 Self-evaluation of 108 trainee nurses

\begin{tabular}{|c|c|c|c|c|c|}
\hline Project & $\begin{array}{l}\text { Significantly } \\
\text { agree, n (\%) }\end{array}$ & $\begin{array}{l}\text { Agree, } \\
\mathrm{n}(\%)\end{array}$ & $\begin{array}{l}\text { Not sure, } \\
\mathrm{n}(\%)\end{array}$ & $\begin{array}{l}\text { Disagree, } \\
\mathrm{n}(\%)\end{array}$ & $\begin{array}{c}\text { Significantly } \\
\text { disagree, n (\%) }\end{array}$ \\
\hline Psychological stress reduction & $80(74.07)$ & $21(19.44)$ & $4(3.70)$ & $1(0.93)$ & $2(1.85)$ \\
\hline $\begin{array}{l}\text { Improved ability to resolve emergencies in a } \\
\text { timely and effective manner }\end{array}$ & $89(82.41)$ & $15(13.89)$ & $1(0.93)$ & $2(1.85)$ & $1(0.93)$ \\
\hline Improved ability to analyze and solve problems & $86(79.63)$ & $12(11.11)$ & $2(1.85)$ & $5(4.63)$ & $3(2.78)$ \\
\hline Critical thinking skills are cultivated & $88(81.48)$ & $17(15.74)$ & $2(1.85)$ & $0(0.00)$ & $1(0.93)$ \\
\hline Increased interest in learning & $87(80.56)$ & $18(16.67)$ & $1(0.93)$ & $1(0.93)$ & $1(0.93)$ \\
\hline Harmonious relationship with colleagues & 90 (83.33) & $12(11.11)$ & $1(0.93)$ & $2(1.85)$ & $3(2.78)$ \\
\hline
\end{tabular}

Evaluation, Input Evaluation, Process Evaluation, Product Evaluation) teaching evaluation system $(4,5)$, which involves aspects such as necessity of simulated teaching, teaching goals, teaching staff, student preparation, case design, situation realization degree, feedback, and teaching satisfaction, etc. Separate questionnaires were the designed for both instructors and trainee nurses. The questionnaires were filled out after each course, and the design and development of the course was subsequently adjusted based on the feedback from the questionnaires.

At the same time, a questionnaire survey was issued to evaluate the nurses' clinical thinking ability, including their critical thinking ability, systemic thinking ability, and evidence-based thinking ability (6). Moreover, a cooperation measurement table was designed for the nursing team $(7,8)$, which involved aspects such as trust, team orientation, support, shared mental model, and team leadership. The theoretical and clinical practice ability of the nurses was also assessed (9).

\section{Statistical analysis}

GraphPad Prism 8 from GraphPad Software (San Diego, California, USA) was used to analyze the relevant data in the study, and the $t$-test was used to perform statistical analysis between the two groups. $\mathrm{P}<0.05$ indicated statistically significant differences in the data.

\section{Results}

\section{Self-evaluation of nurses after receiving emergency training}

A total of 108 questionnaires were distributed following the teaching training, and 108 questionnaires were recovered (recovery rate of $100 \%$ ). The data is shown in Table 1. The survey results showed that in situ simulation teaching was very helpful in the training and education of trainee nurses.

\section{Instructor's evaluation of CIPP teaching}

A total of 108 questionnaires were distributed following the teaching training, and 108 questionnaires were recovered (recovery rate of 100\%). The data is shown in Table 2.

\section{Questionnaire survey of nurses' clinical thinking ability}

A metric table was used to investigate the clinical thinking ability of medical students and the effectiveness of in situ simulation teaching $(6,10)$. The test was conducted prior to undergoing the simulated teaching course and upon completion of the course. By comparing the clinical thinking ability of the trainee nurse with a nurse who has worked for more than 5 years or a nurse with a higher title, a quantitative evaluation of indicators and data analysis was performed. In this evaluation, the full score was 120 , which was converted into a 100 -point system. A score of 80 100 indicated significantly good clinical thinking ability, 60-80 signified good clinical thinking, 40-60 represented general clinical thinking, 20-40 denoted poor clinical thinking, and 0-20 indicated significantly poor clinical thinking ability. The results of the study showed that compared with the control group, the clinical thinking ability of the trainee nurses was improved to a certain extent 
Table 2 Instructor's evaluation of 108 intern nurses

\begin{tabular}{|c|c|c|c|c|c|}
\hline Project & $\begin{array}{l}\text { Significantly } \\
\text { agree, n (\%) }\end{array}$ & $\begin{array}{l}\text { Agree, } \\
\mathrm{n}(\%)\end{array}$ & $\begin{array}{l}\text { Not sure, } \\
\mathrm{n}(\%)\end{array}$ & $\begin{array}{l}\text { Disagree, } \\
\mathrm{n}(\%)\end{array}$ & $\begin{array}{l}\text { Significantly } \\
\text { disagree, n (\%) }\end{array}$ \\
\hline Find problems in time and notify the doctor & $75(69.44)$ & $25(23.15)$ & $3(2.78)$ & $2(1.85)$ & $3(2.78)$ \\
\hline Assist the doctor to solve difficult problems & $78(72.22)$ & $18(16.67)$ & $2(1.85)$ & $8(7.41)$ & $2(1.85)$ \\
\hline $\begin{array}{l}\text { Excellent professional knowledge, skills, and } \\
\text { cooperation with doctors }\end{array}$ & $81(75)$ & $12(11.11)$ & $2(1.85)$ & $5(4.63)$ & $8(7.41)$ \\
\hline $\begin{array}{l}\text { Effectively coordinate the relationship between doctors } \\
\text { and nurses, as well as inter-departmental relationships }\end{array}$ & $85(78.70)$ & $18(16.67)$ & $2(1.85)$ & $2(1.85)$ & $1(0.93)$ \\
\hline Better handle emergency & $87(80.56)$ & $17(15.74)$ & $1(0.93)$ & $1(0.93)$ & $2(1.85)$ \\
\hline No complaints from patients & $86(79.62)$ & $13(12.04)$ & $3(2.78)$ & $3(2.78)$ & $3(2.78)$ \\
\hline
\end{tabular}

Table 3 Evaluation of nurses' clinical thinking ability

\begin{tabular}{lcc}
\hline Project & Trainee nurse (experiment group) & Advanced nurse (control group) \\
\hline Critical thinking skills & $60.84 \pm 4.24$ & $90.12 \pm 2.15^{\# \# \#}$ \\
Before the simulation course & $80.55 \pm 3.45^{\star *}$ & $92.34 \pm 2.46^{\# \#}$ \\
After the simulation course & & $88.44 \pm 4.25^{\# \# \#}$ \\
System thinking ability & $56.45 \pm 3.79$ & $90.46 \pm 3.24$ \\
Before the simulation course & $83.59 \pm 3.58^{* * *}$ & $89.38 \pm 3.21^{\# \# \#}$ \\
After the simulation course & & $91.54 \pm 3.02^{\#}$ \\
Evidence-based thinking skills & $59.68 \pm 3.89$ & $80.99 \pm 3.71^{* *}$ \\
Before the simulation course & & \\
After the simulation course & & \\
\hline
\end{tabular}

Compared with before in situ simulation teaching, ${ }^{* *} \mathrm{P}<0.01$, ${ }^{* *} \mathrm{P}<0.001$; compared with the control group, ${ }^{\#} \mathrm{P}<0.05,{ }^{\# \#} \mathrm{P}<0.01,{ }^{\# \#} \mathrm{P}<0.001$.

after in situ simulation teaching (Table 3).

\section{Nurse teamwork}

The nursing team cooperation metric table was used to evaluate the degree of team cooperation among the trainee nurses. The metric table included five dimensions (trust, team orientation, support, shared mental model, and team leadership), with a total of 32 items $(11,12)$. Trust, team orientation, support, shared mental model and the team leadership contained seven, eight, six, seven, and four items, respectively.

The survey results showed that compared with the presimulation teaching course, the total score for teamwork among the trainee nurses was $(93.98 \pm 6.12)$ points, and the average score of each dimension (from high to low) was
(3.68 \pm 0.38$)$ for the support dimension, $(3.45 \pm 0.34)$ for the team orientation dimension, $(2.64 \pm 0.41)$ for the team leadership dimension, $(2.58 \pm 0.45)$ for the trust dimension, and $(2.50 \pm 0.25)$ for the shared mental model dimension (Table 4 and Figure 1).

\section{Theoretical and clinical practice capabilities}

The theoretical examination papers were mainly based on the theoretical content involved in the rotation, and were combined with case analysis questions to comprehensively assess the nurses' theoretical application ability. The clinical practice ability test involved three common clinical nursing events in the form of simulated nursing emergency cases. Based on the specific condition of the patients and the general conditions, the nurses were required to 
Table 4 Evaluation of trainee nurses' teamwork

\begin{tabular}{lccc}
\hline Project & Dimension & Before the simulation course & After the simulation course \\
\hline Trust & 7 & $2.10 \pm 0.24$ & $2.58 \pm 0.45$ \\
Team orientation & 8 & $1.85 \pm 0.35$ & $3.45 \pm 0.34^{\star \star}$ \\
Support & 6 & $2.45 \pm 0.25$ & $3.68 \pm 0.38^{\star \star}$ \\
Shared mental model & 7 & $1.58 \pm 0.34$ & $2.50 \pm 0.25^{\star}$ \\
Team leadership & 4 & $1.45 \pm 0.65$ & $2.64 \pm 0.41$ \\
Total score & & $62.21 \pm 5.54$ & $93.98 \pm 6.12^{\star \star}$ \\
\hline
\end{tabular}

Compared with before in situ simulation teaching, ${ }^{*} \mathrm{P}<0.05$, ${ }^{\star \star} \mathrm{P}<0.01$.

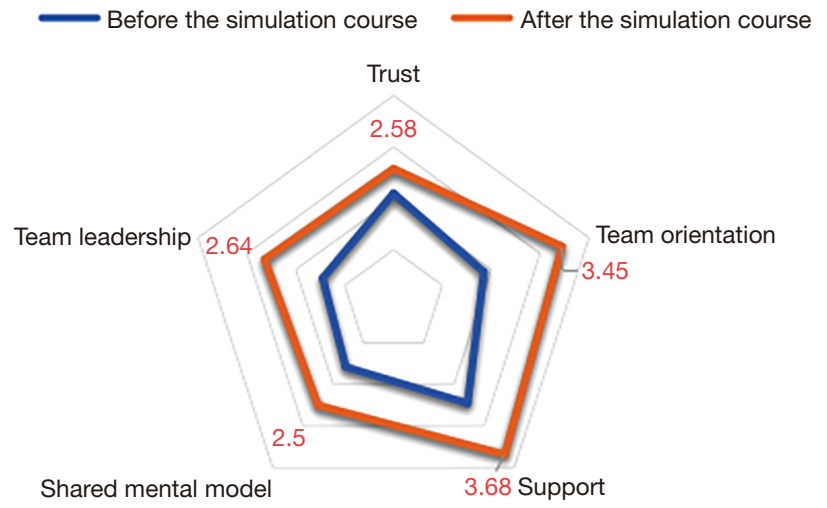

Figure 1 Evaluation of trainee nurses' teamwork.

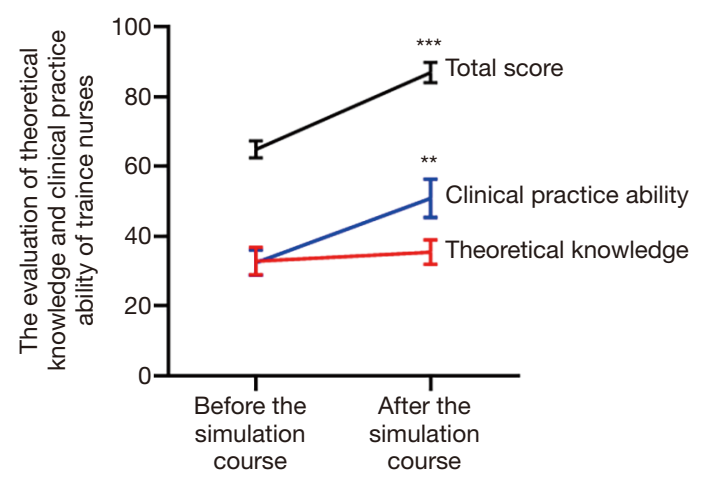

Figure 2 Evaluation of the theoretical and clinical practice ability of trainee nurses (total score of 100 points). Compared with before in situ simulation teaching, ${ }^{* *} \mathrm{P}<0.01,{ }^{* * *} \mathrm{P}<0.001$.

conduct professional assessments of patients, put forward major nursing problems, and propose targeted nursing measures in terms of condition observation, assisted treatment, psychological care, humanistic communication, and education, etc., and evaluate the effectiveness of the nursing measures. Moreover, two common clinical nursing operation techniques were evaluated, and questions were asked on-the-spot. The survey results showed that the clinical practice ability of the trainee nurses was improved to a certain extent after in situ simulation teaching, however there was no difference in theoretical knowledge (Figure 2).

\section{Discussion}

In recent years, with the continuous reform of the hospital system, the demand for high-quality nursing talents has become increasingly urgent. Therefore, the training of relevant nursing staff in responding to nursing emergencies is extremely important. Nursing emergencies refer to nursing-related incidents that are non-routine, unexpected, and sudden (10), including sudden changes in the condition of a patient $(13,14)$, adverse nursing events $(15,16)$, equipment failure $(17)$, and other emergency events. Traditional clinical nursing training is usually a learning process in which the teacher actively teaches and the nurse passively accepts. This approach typically results in nurses having a strong sense of dependence, lack of problem awareness, creative thinking, independence, and poor consciousness. Furthermore, using this method, the enthusiasm for learning among nurses is low, and cannot meet the contemporary demand for nurses (18). In situ simulation teaching to practically train nurses can significantly improve the quality of the nurses' responses to emergencies.

In situ simulation involving placing an ESC simulator in the actual clinical work environment in a real and intuitive way. It provides a way to improve the reliability and safety of high-risk areas, deliberately practicing and integrating team skills in a clinical environment where time pressures exist, which provides an accurate sense of reality. It is also a rich 
resource for identifying potential threats that may endanger patient safety (1). This teaching method offers considerable advantages and value when applied to the education of trainee nurses, such as the authenticity of training, time convenience, case diversity, training adjustability, patient safety, operation error correction, process controllability, teamwork, as well as offering recording and playback functions, etc. In situ simulation teaching has increasingly shown advantages including authenticity, safety, repeatability, high efficiency, and compliance with medical ethics requirements, and is a safe, reliable, and scientific method.

In this emergency training, in situ simulation teaching was employed. Following the training course, the satisfaction of nurses and instructors was evaluated. At the same time, the clinical thinking ability, teamwork of nurses, as well as the theoretical and clinical practice capabilities of the trainee nurses were also assessed. The results showed that the satisfaction rate of in situ simulation teaching for trainee nurses was $94.9 \%$, and the satisfaction of instructors to trainee nurses was $92.2 \%$, indicating that in situ simulation teaching was invaluable to trainee nurses in emergency training.

Nursing clinical thinking refers to the ability of nurses to use theory, intelligence, and experience to comprehensively analyze, judge, make decisions, and implement nursing measures for patients' existing or potential nursing problems. It is a necessary prerequisite and foundation for carrying out holistic nursing according to nursing procedures, and is one of the core abilities that nurses need to possess (19). The survey results showed that the critical thinking, systematic thinking, and evidence-based thinking abilities of trainee nurses were improved to a certain extent, compared with before the in situ simulation teaching. Moreover, compared with experienced nurses, the gap was narrowed, and clinical thinking ability was improved. Therefore, it is necessary to strengthen the training of clinical thinking of trainee nurses to better adapt to clinical nursing work in the future.

In an increasingly complex medical environment, teamwork is particularly crucial to improve the quality of care and maintain patient safety. Studies have confirmed that effective teamwork among nurses can reduce nursing errors, patient mortality, bed falling rates, and improve patient satisfaction. At the same time, it can improve nurses' job satisfaction, increase nursing performance, and promote effective communication between patients, family members, and nurses. Only through continuous and effective teaching and training can the team's emergency collaboration and cohesion be improved (20).

The results of the survey showed that, compared with before the in situ simulation teaching, the nurses' trust, team orientation, support, shared mental models, and team leadership capabilities were all improved. In addition, investigation and analysis of the theoretical and clinical practice abilities indicated that the trainee nurses' clinical practice abilities were greatly improved compared with before the in situ simulation teaching.

In summary, the in situ simulation training method improved the clinical thinking ability, teamwork, as well as the theoretical and clinical practice ability of trainee nurses to a certain extent, and further enhanced their comprehensive abilities, in line with the contemporary requirements for modern nursing talents.

\section{Acknowledgments}

Funding: None.

\section{Footnote}

Reporting Checklist: The authors have completed the MDAR reporting checklist. Available at http://dx.doi.org/10.21037/ apm-21-545

Data Sharing Statement: Available at http://dx.doi. org/10.21037/apm-21-545

Conflicts of Interest: All authors have completed the ICMJE uniform disclosure form (available at http://dx.doi. org/10.21037/apm-21-545). The authors have no conflicts of interest to declare.

Ethical Statement: The authors are accountable for all aspects of the work in ensuring that questions related to the accuracy or integrity of any part of the work are appropriately investigated and resolved. The study was conducted in accordance with the Declaration of Helsinki (as revised in 2013). The study protocol was approved by the ethics committee of the First Affiliated Hospital of Hainan Medical University (No. 20190232). Informed consent was taken from all participants.

Open Access Statement: This is an Open Access article distributed in accordance with the Creative Commons Attribution-NonCommercial-NoDerivs 4.0 International 
License (CC BY-NC-ND 4.0), which permits the noncommercial replication and distribution of the article with the strict proviso that no changes or edits are made and the original work is properly cited (including links to both the formal publication through the relevant DOI and the license). See: https://creativecommons.org/licenses/by-ncnd/4.0/.

\section{References}

1. Shrestha R, Shrestha AP, Shrestha SK, et al. Interdisciplinary in situ simulation-based medical education in the emergency department of a teaching hospital in Nepal. Int J Emerg Med 2019;12:19.

2. Montanaro J. Using In Situ Simulation to Develop a Prone Positioning Protocol for Patients With ARDS. Crit Care Nurse 2020;11:e1-13.

3. Cardinal P, Barton G, DesRosier K, et al. Mapping the Expert Mind: Integration Method for Revising the ACES Medical Simulation Curriculum. J Med Educ Curric Dev 2020;7:2382120520913270.

4. Kool B, Wise MR, Peiris-John R, et.al. Is the delivery of a quality improvement education programme in obstetrics and gynaecology for final year medical students feasible and still effective in a shortened time frame? BMC Med Educ 2017;17:91.

5. Xie J, Zhang T, Cheng F. Construction of Evaluation System for Flipped Classroom Teaching Based on CIPP. Modern Distance Education Research 2017;5:10-2.

6. Brown Tyo M, McCurry MK. An Integrative Review of Clinical Reasoning Teaching Strategies and Outcome Evaluation in Nursing Education. Nurs Educ Perspect 2019;40:11-7.

7. Funk KA, Weaver KK. Team work and collaborative practice agreements among pharmacists and nurse practitioners. J Am Pharm Assoc (2003) 2018;58:117-9.

8. Bragadóttir H, Kalisch BJ, Tryggvadóttir GB. The Extent to which Adequacy of Staffing Predicts Nursing Teamwork in Hospitals. J Clin Nurs 2019;28:4298-309.

9. Alamrani MH, Alammar KA, Alqahtani SS, et al. Comparing the Effects of Simulation-Based and Traditional Teaching Methods on the Critical Thinking Abilities and Self-Confidence of Nursing Students. J Nurs Res 2018;26:152-7.

10. Bogár PZ, Tóth L, Rendeki S, et al. The present and the future of medical simulation education in Hungary. Orv
Hetil 2020;161:1078-87.

11. Aufegger L, Shariq O, Bicknell C, et al. Can shared leadership enhance clinical team management? A systematic review. Leadersh Health Serv (Bradf Engl) 2019;32:309-35.

12. Pedersen TH, Cignacco E, Meuli J, et al. The German interprofessional attitudes scale: translation, cultural adaptation, and validation. GMS J Med Educ 2020;37:Doc32.

13. Hageman JR. Preparation for Emergency Patient Assessment in the Outpatient Setting. Pediatr Ann 2018;47:e87.

14. Yang XR, Fu GN. Application of Scenario Test of Emergent Treatment on Patient in Ability Assessment of Junior Nurses. World Latest Medicine Information 2019;19:338-9.

15. Roberts F, Gettings P, Torbeck L, Helft PR. Reflection on adverse event disclosure in the postsurgical hospital context. J Surg Educ 2015;72:767-70.

16. Ogawa A, Okumura Y, Fujisawa D, et al. Quality of care in hospitalized cancer patients before and after implementation of a systematic prevention program for delirium: the DELTA exploratory trial. Support Care Cancer 2019,27:557-65.

17. Kassam AB, Rovin RA, Walia S, et al. The Operating Room of the Future Versus the Future of the Operating Room. Otolaryngol Clin North Am 2017;50:655-71.

18. Gupta R, Dubé K, Bodenheimer T. The Road to Excellence for Primary Care Resident Teaching Clinics. Acad Med 2016;91:458-61.

19. Schmidt HG, Mamede S. How to improve the teaching of clinical reasoning: a narrative review and a proposal. Med Educ 2015;49:961-73.

20. Spano-Szekely L, Winkler A, Waters C, et al. Individualized Fall Prevention Program in an Acute Care Setting: An Evidence-Based Practice Improvement. J Nurs Care Qual 2019;34:127-32.

(English Language Editor: A. Kassem)

Cite this article as: Wang $\mathrm{W}$, Han $\mathrm{C}$, Zhang $\mathrm{X}$, Tong $\mathrm{Y}$, Zhao R, Wang B, Xing W, Wang N, Chen C. Application of in situ simulation teaching in the training of trainee nurses to respond to emergencies. Ann Palliat Med 2021;10(4):4509-4515. doi: 10.21037/apm-21-545 